\title{
Densidade básica e estabilidade dimensional de madeiras tropicais modificadas por tratamentos térmicos
}

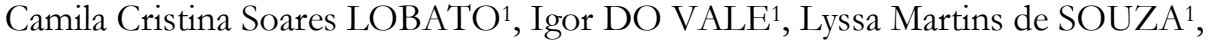 \\ Juliana Livian Lima de ABREU1 ${ }^{1}$, Gracialda Costa FERREIRA ${ }^{1}$, Lina BUFALINO ${ }^{1 *}$
}

${ }^{1}$ Instituto de Ciências Agrárias, Universidade Federal Rural da Amazônia, Belém, PA, Brasil. (Orcid: 0000-0002-1557-0829; 0000-0003-2773-1164; 0000-0002-2206-2275; 0000-0001-7295-7889; 0000-0002-4018-9624; *) *E-mail: linabufalino1@gmail.com (Orcid: 0000-0002-7688-3140)

Recebido em 15/06/2020; Aceito em 27/10/2020; Publicado em 11/11/2020.

\begin{abstract}
RESUMO: O objetivo deste estudo foi avaliar as modificações de propriedades físicas das madeiras de três espécies tropicais de interesse comercial por tratamentos térmicos conduzidos com diferentes temperaturas e tempos de exposição ao calor. Amostras de madeira de Pouteria caimito, Protium altissimum e Jacaranda copaia foram submetidas a modificações térmicas a 180 e $240^{\circ} \mathrm{C}$, ambas por 1 e $2 \mathrm{~h}$. Foram avaliadas redução de massa, densidade básica e contração volumétrica das madeiras. $\mathrm{O}$ tratamento de $2 \mathrm{~h}$ a $240{ }^{\circ} \mathrm{C}$ causou maior redução de massa para todas as espécies. Pouteria caimito e Protium altissimum não apresentaram diferença significativa na densidade básica e contração volumétrica entre os tratamentos térmicos. A redução de massa, que confirma a efetividade dos tratamentos térmicos, aliada à manutenção da densidade é básica, é benéfica para a qualidade das madeiras. Jacaranda copaia apresentou maior estabilidade dimensional, mas foi mais afetada pelas variações dos tratamentos com diminuição da densidade básica da madeira quando submetida a $180{ }^{\circ} \mathrm{C}$ por $1 \mathrm{~h}$ e $240{ }^{\circ} \mathrm{C}$ por $2 \mathrm{~h}$. A secagem das madeiras não tratadas, assim como os tratamentos térmicos, diminui a capacidade de adsorção de água e o volume saturado, resultando em contrações volumétricas similares às das madeiras termicamente tratadas.
\end{abstract}

Palavras-chave: secagem da madeira; hornificação; densidade básica; contração volumétrica.

\section{Basic density and dimensional stability of tropical woods modified by thermal treatments}

\begin{abstract}
This study aimed to evaluate the wood physical properties' modifications from three commercially interesting tropical species by thermal treatments carried out with different temperatures and heat exposure times. Wood samples of Pouteria caimito, Protium altissimum, and Jacaranda copaia were submitted to thermal modifications at 180 and $240{ }^{\circ} \mathrm{C}$, both for 1 and $2 \mathrm{~h}$. The mass reduction, basic density, and volumetric contraction were evaluated. The treatment of $2 \mathrm{~h}$ at $240{ }^{\circ} \mathrm{C}$ caused the highest mass reduction for all species. Pouteria caimito and Protium altissimum did not significantly differ in basic density and volumetric contraction of wood among thermal treatments. The mass reduction, which confirms the effectiveness of thermal treatments, allied to the maintenance of basic density, benefits the wood quality. Jacaranda copaia showed the greatest dimensional stability, but it was the most affected species by the variations of thermal treatments with decreases in basic wood density when submitted to $180^{\circ} \mathrm{C}$ for $1 \mathrm{~h}$ and $240{ }^{\circ} \mathrm{C}$ for $2 \mathrm{~h}$. The drying of the samples, as well as the thermal treatments, may decrease the water adsorption and saturated volume, resulting in volumetric contractions similar to those of thermally treated samples.
\end{abstract}

Keywords: wood drying; hornification; basic density; volumetric contraction.

\section{INTRODUÇÃO}

A região amazônica apresenta a maior diversidade e reserva de espécies arbóreas do mundo (SLIK et al., 2015), o que contribui para seu papel fundamental como fornecedora de madeiras tropicais para os mercados nacional e internacional. As madeiras de espécies tropicais são particularmente atraentes para as indústrias de construção civil, painéis e moveleiras devido aos seus grandes diâmetros, texturas variadas e alta durabilidade e resistência natural. Entretanto, o setor madeireiro local é limitado pela falta de tecnologias de processamento adequadas e de pouca informação sobre as matérias-primas potenciais ainda pouco exploradas (REIS et al., 2015).

O conhecimento das características anatômicas, físicas e mecânicas da madeira é fundamental para possibilitar o uso do material de maneira mais econômica e segura (DE MOURA; DOURADO, 2018). Um dos maiores desafios para profissionais da tecnologia da madeira tem sido encontrar soluções para a instabilidade dimensional e durabilidade da madeira (POUBEL et al., 2013). Tais características podem ser melhoradas com aplicação de tratamentos térmicos.

A modificação térmica de peças de madeira pode ser realizada pela aplicação de temperaturas que podem variar entre 180 e $260{ }^{\circ} \mathrm{C}$ (HILL, 2006). Entretanto, conforme Cademartori et al. (2012), a partir de $200{ }^{\circ} \mathrm{C}$, as modificações nas propriedades físico-mecânicas passam a ser mais significativas. Adicionalmente, o tempo de exposição da madeira ao calor também varia na literatura. Mendes et al. (2013) diminuíram o caráter hidrofílico da madeira de Pinus 
taeda aplicando tratamentos térmicos de 200 e $240^{\circ} \mathrm{C}$ por 1 h. Já Gunduz et al. (2009) utilizaram uma temperatura de $160^{\circ} \mathrm{C}$ por um período de exposição ao calor mais longo de $2 \mathrm{~h}$ para melhorar a estabilidade dimensional da madeira de Pyrus elaeagnifolia.

Este processo vem sendo desenvolvido na Europa nos últimos 20 anos e já é adotado em escala industrial em vários países, como Finlândia, (ThermoWood $\AA)$, França (Retification $\AA$ e Le Bois Perdure $\AA)$, Holanda (PlatoProcess ${ }^{\circledR}$ ) e Alemanha (Oil-Heat-Treatment) (POUBEL et al., 2013). As principais diferenças entre esses tratamentos são a temperatura máxima aplicada, tempo de tratamento, uso ou não de atmosfera inerte com nitrogênio ou com vapor, uso de óleos e/ou se o processo é aplicado para madeira seca ou úmida (HILL, 2006).

No Brasil, as pesquisas sobre modificação térmica ainda são pouco exploradas quando comparadas a outros processos de industrialização da madeira, tais como desdobro, secagem, polpação, carbonização e produção de painéis (BATISTA, 2019). Além disso, grande parte dessas pesquisas são realizadas em madeiras exóticas utilizadas em reflorestamentos, como as do gênero Eucalyptus e Pinus, já que as mesmas são abundantes em plantios comerciais (DELUCIS et al., 2014). Existe, portanto, uma lacuna de conhecimento para espécies tropicais da região amazônica, que permanecem com os seus potenciais tecnológicos madeireiros ainda pouco empreendidos. Neste contexto, o objetivo deste estudo é avaliar as propriedades físicas da madeira proveniente de três espécies tropicais de interesse comercial após tratamentos com modificação térmica realizados com variações na temperatura e tempo de exposição.

\section{MATERIAL E MÉTODOS}

As amostras foram coletadas no pátio de estocagem de madeira oriundo de supressão vegetal em área de mineração $\left(-3.246735^{\circ},-47.737292^{\circ}\right)$ no município de Paragominas, nordeste do Pará. Foram selecionados indivíduos pertencentes a três espécies tropicais: Pouteria caimito (abiu), Protium altissimum (breu) e Jacaranda copaia (parapará). Os critérios de seleção foram interesse comercial, ampla disponibilidade na área e densidade básica, sendo preferidas espécies com densidades básicas diferentes de acordo com a descrição da empresa, pois esta propriedade pode interferir no comportamento da madeira. A identificação das espécies foi realizada por parabotânicos na xiloteca da Embrapa Amazônia Oriental.

Para cada espécie, uma tora foi selecionada para a retirada com motosserra de um disco de madeira com diâmetro de aproximadamente $80 \mathrm{~cm}$. Cada disco foi armazenado em saco plástico e transportado para o laboratório onde foram realizados os cortes das amostras, seguidos dos tratamentos térmicos e análises das propriedades físicas da madeira (Figura 1).

Os discos foram cortados com o auxílio de uma serra circular em cunhas opostas passando pela medula. Duas delas foram destinadas à obtenção de 20 corpos de prova por espécie, sendo 10 corpos de prova por cunha, dispostos em duas fileiras tangenciais de 5 corpos de prova cada e localizadas no alburno. O corte de uma das dimensões $(2,0$ $\mathrm{cm})$ foi realizado acompanhando um raio do disco em vista transversal. Um total de 60 amostras (20 para cada espécie), com dimensões de 3,0 × 2,0 × 5,0 cm nas direções tangencial, radial e axial, respectivamente, foram obtidas conforme as diretrizes estabelecidas pela NBR 7190/97 (ABNT, 1997). Para cada espécie, as 20 amostras foram distribuídas para os cinco tratamentos, resultando em 4 repetições por tratamento.

Os tratamentos térmicos dos corpos de prova foram realizados em atmosfera oxidativa em um forno elétrico do tipo mufla Lab1000®, com variação de temperatura e tempo de exposição ao calor (Tabela 1), estipulados conforme a literatura (HILL, 2006; GUNDUZ et al., 2009; MENDES et al., 2013). Os tempos de aquecimento, de aproximadamente $25 \mathrm{~min}$ e $50 \mathrm{~min}$, foram necessários para atingir respectivamente a menor $\left(180{ }^{\circ} \mathrm{C}\right)$ e maior $\left(240{ }^{\circ} \mathrm{C}\right)$ temperatura de patamar utilizadas no estudo. Ao atingir a temperatura máxima de patamar, a mesma foi mantida por 1 ou 2 h. Em seguida, a mufla foi desligada e mantida fechada. Os corpos de prova foram retirados quando o equipamento atingiu temperatura ambiente. Para cada tratamento térmico foram utilizados quatro corpos de provas de cada espécie. As propriedades da madeira analisadas foram redução de massa, densidade básica e contração volumétrica máxima.

Tabela 1. Tratamentos térmicos aplicados às madeiras de diferentes espécies tropicais.

Table 1. Thermal treatments applied to the woods from different tropical species.

\begin{tabular}{ccc}
\hline Tratamento & $\begin{array}{c}\text { Temperatura máxima de } \\
\text { patamar }\left({ }^{\circ} \mathrm{C}\right)\end{array}$ & $\begin{array}{c}\text { Tempo de permanência } \\
\text { na mufla }(\mathrm{h})\end{array}$ \\
\hline $180 / 1$ & 180 & 1 \\
$180 / 2$ & 180 & 2 \\
$240 / 1$ & 240 & 1 \\
$240 / 2$ & 240 & 2 \\
Ctrl & Controle & Controle \\
\hline
\end{tabular}

Antes dos tratamentos térmicos, todos os corpos de prova foram submetidos à secagem a $105 \pm 3^{\circ} \mathrm{C}$ em estufa até remoção completa de água (aproximadamente $24 \mathrm{~h}$ ), estipulada por massa constante das amostras após 3 pesagens consecutivas, caracterizando umidade de $0 \%$. Ao final da secagem, os corpos de prova foram resfriados em dessecador para obtenção da massa absolutamente seca, que corresponde à massa antes do tratamento térmico (M1).

Após os tratamentos térmicos, os corpos de prova foram novamente pesados para obtenção da massa após modificação térmica (M2). Assim, a redução de massa decorrente do tratamento térmico foi calculada de acordo com a Equação (1) (PONCSÁK et al., 2006).

$$
\mathrm{RM}=\frac{(\mathrm{M} 1-\mathrm{M} 2)}{\mathrm{M} 1} \mathrm{X} 100
$$

em que: $\mathrm{RM}=$ redução de massa (\%); M1= massa seca antes da modificação térmica $(\mathrm{g})$; e M2 = massa seca após modificação térmica $(\mathrm{g})$.

A densidade da madeira foi determinada com base na NBR 11941 adaptada (ABNT, 2003), já que a massa foi determinada antes do volume, pois foi necessário obter a massa absolutamente seca (M1) para o cálculo da redução de massa. Os corpos de prova das madeiras após os tratamentos térmicos e os do controle foram pesados para determinar a massa absolutamente seca (M1). Em seguida, foram imersos em dessecador contendo água destilada e submetidos a ação de uma bomba a vácuo até expansão máxima detectada por mensurações seguidas que não indicaram modificação do volume das amostras. O volume dos corpos de prova foi 
obtido pelo método do princípio de Arquimedes. Assim, o cálculo da densidade básica da madeira foi realizado pela Equação (2):

$$
\mathrm{DB}=\mathrm{M} 1 / \mathrm{Vs}
$$

em que: $\mathrm{DB}=$ Densidade básica $\left(\mathrm{g} / \mathrm{cm}^{3}\right) ; \mathrm{M} 1=$ Massa absolutamente seca (g); e Vs = Volume da madeira acima do PSF $\left(\mathrm{cm}^{3}\right)$.

O volume da madeira absolutamente seca (V0) também foi determinado pelo princípio de Arquimedes para o cálculo da contração volumétrica máxima ( $\beta \mathrm{Vmax})$, obtido pela Equação (3):

$$
\beta \operatorname{Vmax}=((\mathrm{Vs}-\mathrm{V} 0) / \mathrm{Vs}) \times 100
$$

em que: $\beta V \max =$ contração volumétrica máxima $(\%) ; \mathrm{Vs}=$ volume da madeira acima do PSF $\left(\mathrm{cm}^{3}\right) ;$ e $\mathrm{V0}=$ volume da madeira absolutamente seca $\left(\mathrm{cm}^{3}\right)$.

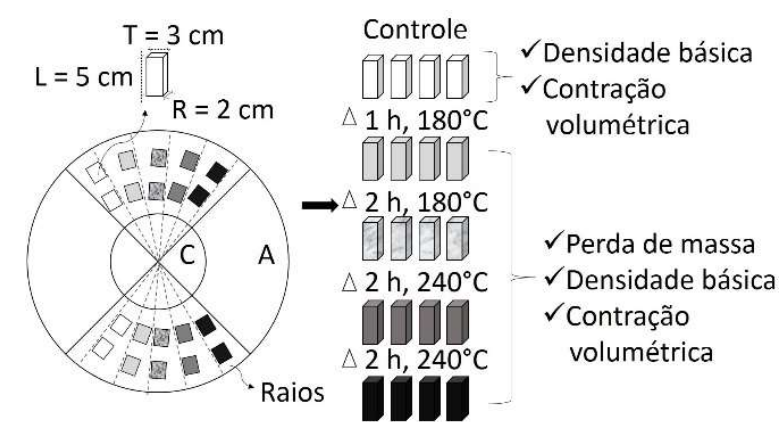

Figura 1. Esquema da amostragem dos corpos de prova distribuídos no disco de madeira e para os diferentes tratamentos térmicos (conforme Tabela 1) e análises conduzidas: $\mathrm{T}$ - eixo tangencial; $\mathrm{R}$ - eixo radial; $\mathrm{L}$ - eixo longitudinal; $\mathrm{C}$ - cerne; e A - alburno.

Figure 1. Scheme of the specimen sampling distributed in the wood disk and for to the different thermal treatments (according to Table 1 ) and conducted analyses: $\mathrm{T}$ - tangential axis; $\mathrm{R}$ - radial axis; L - longitudinal axis; $\mathrm{C}$ - heartwood; and A - sapwood.

O teste não-paramétrico de Kruskal-Wallis foi usado para comparar a redução de massa, densidade básica e a contração volumétrica máxima entre os tratamentos, pois os dados não apresentaram normalidade. O teste post hoc de ConoverIman foi utilizado para comparar os tratamentos em pares. Foi considerada a probabilidade de 0,05 para a significância dos testes. Foram utilizados os pacotes stats e PMCMR da plataforma R (R Foundation for Statistical Computing, Vienna, AT).

\section{RESULTADOS}

A redução de massa foi a única propriedade física que mostrou variação entre os tratamentos térmicos para todas as espécies; enquanto que para densidade básica e contração volumétrica máxima, somente Jacaranda copaia apresentou diferenças significativas entre os tratamentos térmicos aplicados.

Houve maior redução de massa nos tratamentos de maior temperatura $\left(240^{\circ} \mathrm{C}\right)$ para as madeiras de todas as espécies testadas. Além da influência da temperatura, Pouteria caimito $\left(\mathrm{X}^{2}(3)=9,97, \mathrm{p}<0,05\right)$ e Protium altissimum $\left(\mathrm{X}^{2}(3)=9,67, \mathrm{p}\right.$ $<0,05)$ mostraram ainda maior redução de massa com tratamentos de $2 \mathrm{~h}$ na mufla a $240{ }^{\circ} \mathrm{C}$ (Figura 2).
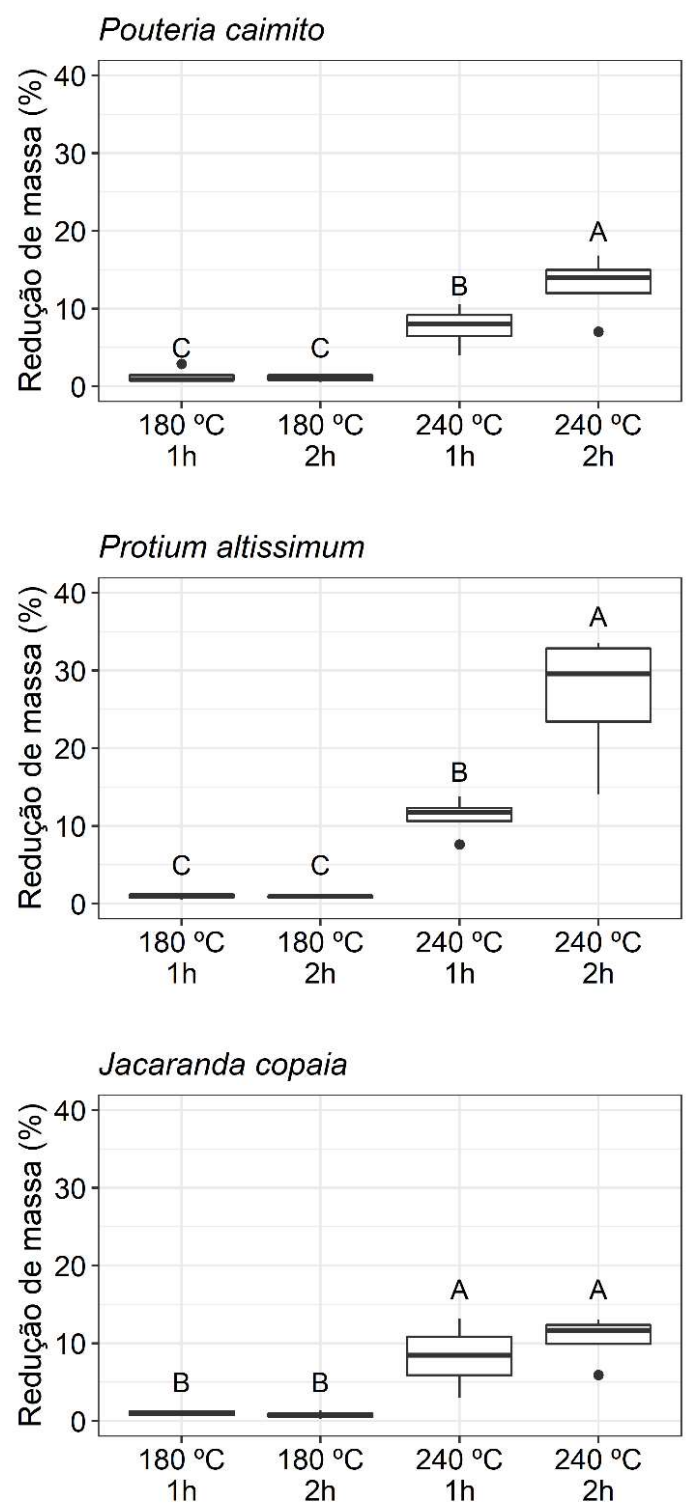

Figura 2. Box-plot da redução de massa da madeira de três madeiras tropicais sob diferentes tratamentos térmicos (conforme a Tabela 1). Letras diferentes indicam diferenças estatísticas entre os tratamentos (Teste de Kruskal-Wallis; $\mathrm{p}<0,05$ ).

Figure 2. Box-plot of the mass reduction of the wood of four tropical woods under different thermal treatments (according to Table 1). Different letters indicate statistical differences among treatments (Kruskal-Wallis test, $\mathrm{p}<0.05$ ).

As densidades básicas das madeiras de Pouteria caimito e Protium altissimum não apresentaram diferença significativa entre os tratamentos. Jacaranda copaia mostrou menor densidade básica para os tratamentos a $180{ }^{\circ} \mathrm{C}$ por $1 \mathrm{~h}$ e 240 ${ }^{\circ} \mathrm{C}$ por $2 \mathrm{~h}$, sem diferença entre os demais tratamentos $\left(\mathrm{X}^{2}\right.$ (4) $=10,13, \mathrm{p}<0,05)($ Figura 3).

As contrações volumétricas máximas das madeiras de Pouteria caimito e Protium altissimum não foram estatisticamente diferentes entre os tratamentos térmicos e os controles. A Jacaranda copaia foi a única espécie que apresentou diferença significativa entre os tratamentos, com menor contração volumétrica máxima para o tratamento a $240{ }^{\circ} \mathrm{C}$ por $2 \mathrm{~h}$ na mufla, e maior contração volumétrica para amostras a $240{ }^{\circ} \mathrm{C}$ por $1 \mathrm{~h}\left(\mathrm{X}^{2}(4)=10,3, \mathrm{p}<0,05\right)$ (Figura 4). 


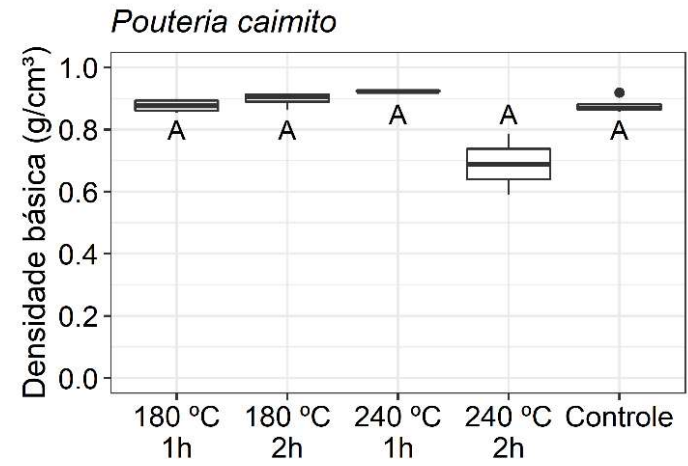

Protium altissimum
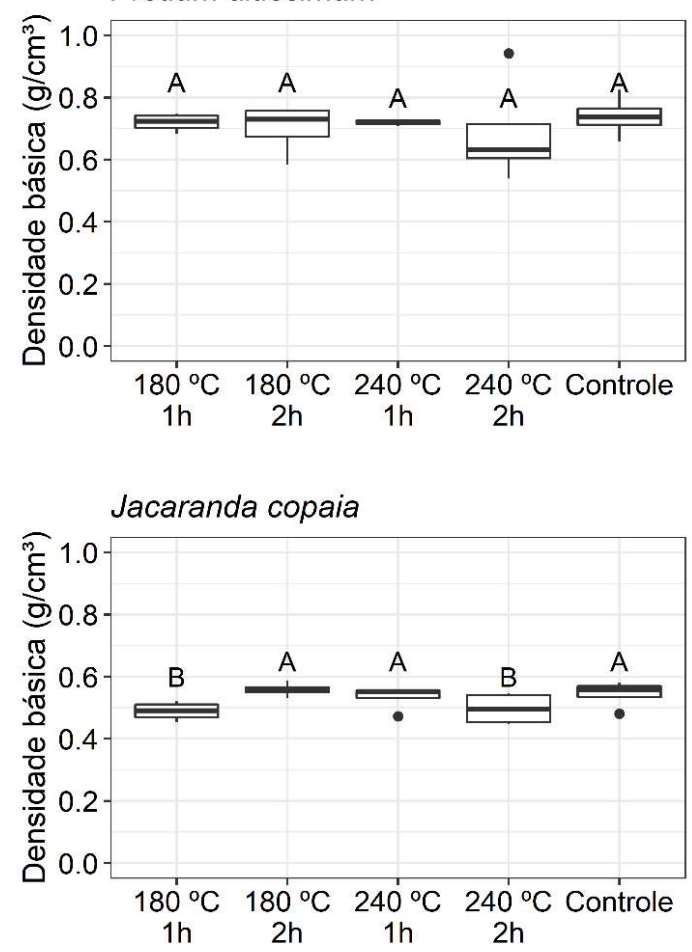

Figura 3. Box-plot da densidade básica da madeira de três madeiras tropicais sob diferentes tratamentos térmicos (conforme a Tabela 1). Letras diferentes indicam diferenças estatísticas entre os tratamentos (Teste de Kruskal-Wallis; $\mathrm{p}<0,05$ ).

Figure 3. Box-plot of the basic wood density of four tropical woods under different thermal treatments (according to Table 1). Different letters indicate statistical differences among treatments (KruskalWallis test, $\mathrm{p}<0.05)$.

\section{DISCUSSÃO}

A maior redução de massa observada nas madeiras de todas as espécies submetidas a temperaturas mais altas se deve à elevada degradação de parte de seus constituintes químicos como extrativos, celulose e hemiceluloses (BRITO et al., 2008; LENGOWSKI et al., 2018). As altas temperaturas afetam principalmente hemiceluloses, termicamente instáveis, e as regiões amorfas da celulose, que são despolimerizadas durante a modificação térmica (ESTEVES et al., 2007; LENGOWSKI et al., 2018). Enquanto a degradação térmica das hemiceluloses ocorre entre 160 a $260{ }^{\circ} \mathrm{C}$, a degradação das celuloses ocorre a temperaturas mais altas, de 200 a $280{ }^{\circ} \mathrm{C}$ (FIGUEROA; DE MORAES, 2009), ou seja, os tratamentos a $240{ }^{\circ} \mathrm{C}$, comparados aos de $180{ }^{\circ} \mathrm{C}$, conseguem reduzir esses carboidratos em maior escala.
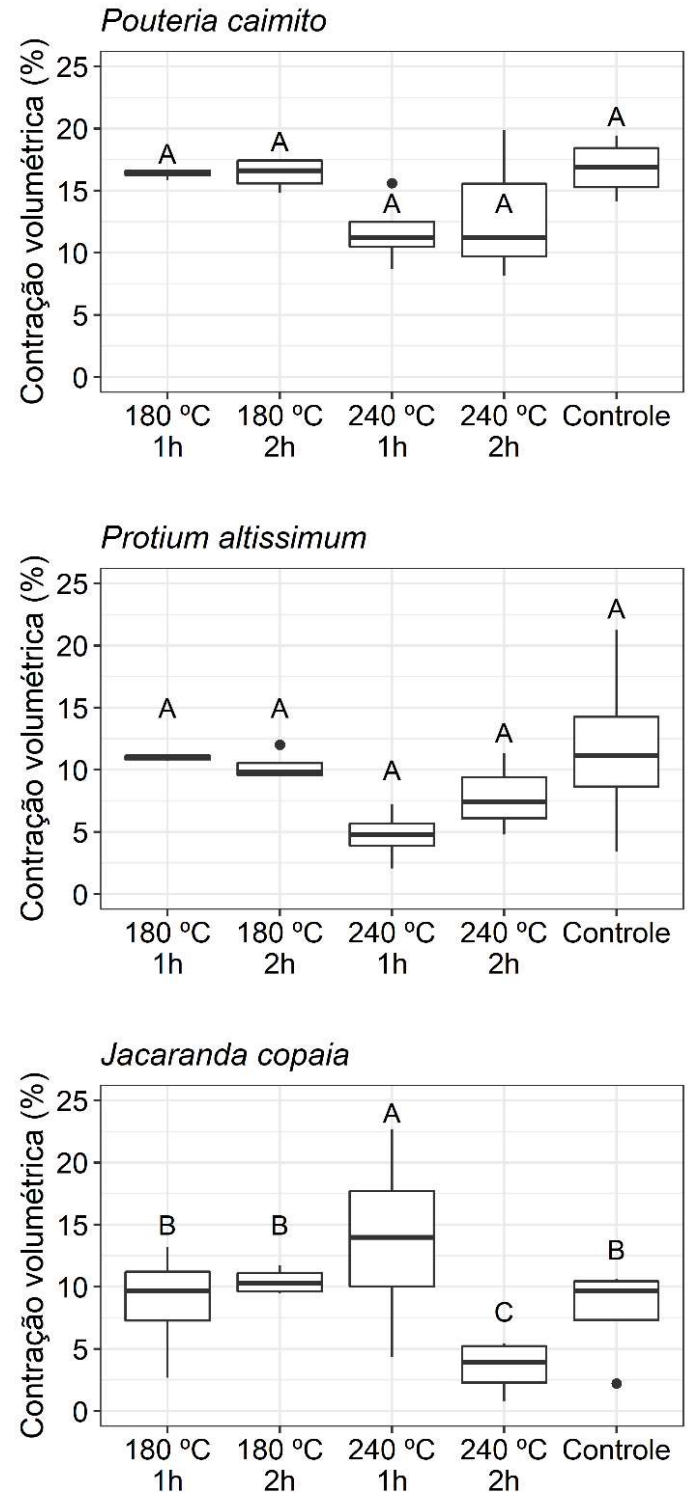

Figura 4. Box-plot da contração volumétrica máxima da madeira de três espécies tropicais sob diferentes tratamentos térmicos (conforme a Tabela 1). Letras diferentes indicam diferenças estatísticas entre os tratamentos (Teste de Kruskal-Wallis; $\mathrm{p}<0,05$ ). Figure 4. Box-plot of the maximum volumetric contraction of the wood of four tropical species under different thermal treatments (according to Table 1). Different letters indicate statistical differences among treatments (Kruskal-Wallis test, $\mathrm{p}<0.05$ ).

A lignina, por outro lado, é termicamente mais resistente que os carboidratos que compõem a madeira (WATKINS et al., 2015). Seu teor pode permanecer igual ou aumentar na madeira pós modificação térmica devido à perda dos demais componentes (LENGOWSKI et al., 2018). Contudo, em torno de $200{ }^{\circ} \mathrm{C}$, ocorrem reações de desidratação da lignina, o que também pode ter influenciado na maior redução de massa nos tratamentos a $240{ }^{\circ} \mathrm{C}$ (FIGUEROA; de MORAES, 2009).

As madeiras deste trabalho foram submetidas a tratamentos térmicos após secagem. Portanto, a perda de massa foi causada pela degradação térmica de parte de seus constituintes químicos. Foi comprovado que as modificações químicas dos polímeros da parede celular das células por tratamentos térmicos foram diretamente responsáveis pela melhoria da durabilidade da madeira à biodeterioração. Os 
mecanismos relacionados à esta melhoria são: 1) aumento da hidrofobicidade que causa ambiente desfavorável ao desenvolvimento de fungos; 2) modificação da estrutura química de extrativos que podem se tornar fungicidas; 3) modificações estruturais que dos polímeros, tornando-os irreconhecíveis para as enzimas de fungos; e 4) degradação de hemiceluloses, que são nutritivas para muitos agentes xilófagos (CHAOUCH et al., 2013). Os autores ressaltaram que todas as madeiras com degradação acima de $10 \%$ se tornaram mais resistentes à biodeterioração. Portanto, os tratamentos térmicos a $240^{\circ} \mathrm{C}$ deste estudo foram eficazes em reduzir as massas das três madeiras amazônicas de interesse comercial e, possivelmente, têm potencial de melhorar sua resistência biodeterioração.

Apesar da maior temperatura ocasionar maior redução de massa em todas as espécies, o efeito do tempo de exposição às diferentes temperaturas não foi similar entre as madeiras testadas. Apenas Pouteria caimito e Protium altissimum mostraram maior redução de massa nos tratamentos a $240{ }^{\circ} \mathrm{C}$ por 2 horas em relação aos tratamentos a $240^{\circ} \mathrm{C}$ por apenas 1 hora. Mesmo tendo mostrado tendência de maior redução de massa ao longo do tempo de exposição, Jacaranda copaia não apresentou diferença significativa em relação ao tempo nos tratamentos a $240{ }^{\circ} \mathrm{C}$, o que pode estar relacionado com a sua menor densidade em comparação com as outras duas espécies (STALLBAUN et al., 2017). Portanto, por apresentar madeira de menor densidade (menor proporção de massa por volume) em relação às outras espécies, Jacaranda copaia tem mais massa reduzida em menor tempo. Após $1 \mathrm{~h}$, é possível que a taxa de redução da massa se torne não significativa. Conforme o tempo de exposição ao calor vai aumentando, a densidade das madeiras passa a ter menos influência do que a química da madeira (CHAOUCH et al., 2013).

Assim, esperava-se que madeiras de menor densidade básica tivessem maior redução percentual de massa quando submetidas a tratamentos térmicos, pois as mesmas têm menos massa por unidade de volume. Entretanto, neste trabalho, Protium altissimum de maior densidade do que Jacaranda copaia, apresentou notável redução de massa a $240^{\circ} \mathrm{C}$ por $2 \mathrm{~h}$, o que pode estar relacionada à sua composição química, especialmente alto teor de extrativos, acima de 10\% (LIMA, 2020), que se degradam a temperaturas mais baixas do que celulose, hemiceluloses e lignina.

Jacaranda copaia também foi a única espécie que mostrou diferença estatística entre os tratamentos térmicos quanto a densidade básica, contudo teve comportamento irregular, com menor densidade para os tratamentos a $180{ }^{\circ} \mathrm{C}$ por $1 \mathrm{~h} \mathrm{e}$ a $240{ }^{\circ} \mathrm{C}$ por $2 \mathrm{~h}$. A diminuição da densidade básica era esperada para as madeiras que passaram por tratamentos devido à redução de massa que ocorre com a modificação térmica. Entretanto, nos demais tratamentos de Jacaranda copaia, e em todos os tratamentos de Pouteria caimito e Protium altissimum, a densidade básica foi similar aos controles e não houve diferença significativa entre os tratamentos térmicos e controle.

Para entender esses resultados, é necessário analisar o processo de perda de água na madeira desde a derrubada da árvore até a determinação da densidade básica. A madeira em seu estado natural, ou seja, sem nunca ter sido submetida à secagem, apresenta, logo após derrubada da árvore, paredes celulares completamente saturadas com água de adesão que se liga às hidroxilas livres dos seus constituintes químicos (GLASS; ZELINKA, 2010). Durante a secagem, a estrutura da parede celular gradualmente colapsa conforme a água é removida e as microfibrilas de celulose se aproximam fazendo ligações de hidrogênio entre si. Estas alterações podem ser irreversíveis, já que muitas das hidroxilas ficam indisponíveis para reidratação completa da madeira (SUCHY et al., 2010; THYBRING et al., 2017). Este fenômeno, chamado de hornificação (THYBRING et al., 2017), provavelmente ocorreu durante secagem realizada a $105 \pm$ $3^{\circ} \mathrm{C}$ para a determinação da massa absolutamente seca de todas amostras, inclusive das amostras controles. Aquelas submetidas aos tratamentos térmicos, em seguida, provavelmente tiveram esse fenômeno intensificado. Após determinação da massa absolutamente seca, as amostras foram submetidas à saturação para determinar seus volumes.

A explicação dos valores de densidade básica terem se mantido, portanto, estaria na diminuição da capacidade de reidratação da madeira após a primeira secagem e os tratamentos térmicos, reduzindo o volume máximo (volume verde) a ser atingido pela madeira por saturação e na diminuição proporcional da massa por degradação térmica de seus constituintes (HILL, 2006).

É importante ressaltar ainda que a água de adesão que causa a expansão da madeira, se liga às hidroxilas livres das hemiceluloses e às regiões amorfas da celulose (THYBRING et al., 2017), já a lignina, além de termicamente mais estável, tem uma quantidade baixa de hidroxilas livres. Visto que a redução de massa foi confirmada, devido à degradação e volatilização parciais dos carboidratos durante o tratamento térmico que resultou em menores grupos de sítios de ligação para a água de adesão, o volume máximo alcançado pela madeira saturada sofreu influência da perda de hemiceluloses, além da hornificação da celulose (HILL, 2006).

Assim como ocorreu para a densidade básica, os resultados de retratibilidade podem ser explicados pelos efeitos de hornificação nos controles e nas amostras pós modificação térmica, além da degradação das hemiceluloses para o segundo tipo. A contração máxima aqui apresentada é a medida da variação dimensional das amostras no seu volume saturado máximo até seu o volume mínimo $(0 \%)$. Dessa forma, ficou evidente que a secagem até umidade a $0 \%$ dos controles ocasionou o mesmo efeito de hornificação que os tratamentos térmicos, impedindo modificações expressivas de estabilidade dimensional (HILL, 2006). Portanto, os tratamentos térmicos não resultaram em diminuição significativa da retratibilidade para as madeiras de Protium altissimum e Pouteria caimito, em comparação com as amostras secas a $100^{\circ} \mathrm{C}$ até massa constante.

Apesar dos efeitos de hornificação ocorridos nas demais amostras, a madeira de Jacaranda copaia após modificação térmica a $240{ }^{\circ} \mathrm{C}$ por $2 \mathrm{~h}$ resultou em maior diminuição da contração volumétrica da madeira, como esperado. Características da madeira como anisotropia e instabilidade dimensional com variações da umidade de equilíbrio higroscópica que, por sua vez, depende de variações da temperatura e umidade do ambiente, limitam suas aplicações. As expansões e contrações devido á entrada e saída de água de adesão, podem causar defeitos como rachaduras, biodeterioração e transformações da superfície, diminuindo o tempo de vida da peça de madeira. Portanto, reduzir a instabilidade dimensional por meio de tratamentos térmicos melhora a qualidade da madeira para construção civil, movelaria, decoração e outras aplicações (HE et al., 2019). 


\section{CONCLUSÕES}

Os tratamentos térmicos foram eficientes na redução de massa de todas as espécies com potencial de contribuir para o aumento da resistência biológica das madeiras. Para Pouteria caimito e Protium altissimum, a manutenção da densidade básica aliada à maior perda de massa a $240^{\circ} \mathrm{C}$ por $2 \mathrm{~h}$ é vantajosa. Nessa mesma condição, Jacaranda copaia apresentou melhoria na estabilidade dimensional, mas diminuição da densidade básica. Tanto os tratamentos térmicos quanto a secagem a $100^{\circ} \mathrm{C}$ diminuem a expansão total da madeira, o que resultou em similaridades entre as propriedades físicas das amostras submetidas a tratamentos térmicos ou secagem até $0 \%$ de umidade.

\section{AGRADECIMENTOS}

Este estudo recebeu apoio técnico e financeiro da Empresa Norsk Hydro, que concedeu as amostras de madeiras utilizadas no estudo; e do governo brasileiro através da Coordenação de Aperfeiçoamento de Pessoal de Nível Superior (CAPES) que concedeu bolsa de estudo a Camila Lobato.

\section{REFERÊNCIAS}

ASSOCIAÇÃO BRASILEIRA DE NORMAS TÉCNICAS. NBR 7190: Projeto de estruturas de madeira. Rio de Janeiro: ABNT, 1997. 107p.

ASSOCIAC ÃO BRASILEIRA DE NORMAS TÉCNICAS. NBR 14660: Madeira- Determinação de densidade básica. Rio de Janeiro: ABNT. 2003, 7p.

BATISTA, D. C. Retificação térmica, termorretificação, tratamento térmico, tratamento com calor ou modificação térmica? Ciência Florestal, Santa Maria, v. 29, n. $1, \quad$ p. 463-480, 2019. DOI: https://doi.org/10.5902/1980509822577

BRITO, J. O.; SILVA, F. G.; LEÃO, M. M.; ALMEIDA, G. Chemical composition changes in eucalyptus and pinus woods submitted to heat treatment. Bioresource Technology, Essex, v. 99, n. 18, p. 8545-8548, out. 2008. DOI: https://doi.org/10.1016/j.biortech.2008.03.069

CADEMARTORI, P. H. G. D.; SCHNEID, E.; GATTO, D. A.; BELTRAME, R.; STANGERLIN, D. M. Modification of static bending strength properties of Eucalyptus grandis heat-treated wood. Materials Research, São Carlos, v. 15, n. 6, p. 922-927, 2012. Doi: https://doi.org/10.1590/S1516-14392012005000136

CHAOUCH, M.; DUMARÇAY, S.; PÉTRISSANS, A.; GÉRARDIN, P. Effect of heat treatment intensity on some conferred properties of different European softwood and hardwood species. Wood Science and Technology, New York, v. 47, p. 663-673, 2013. DOI: https://doi.org/10.1007/s00226-013-0533-z

DE MOURA, M. F. S. F.; DOURADO, N. Wood Fracture Characterization. 1 ed. Boca Raton: CRC Press Taylor \& Francis Group, 2018. 137p.

DELUCIS, R. A.; GATTO, D. A.; CADEMARTORI, P. H. G.; MISSIO, A. L.; SCHNEID, E. Propriedades físicas da madeira termorretificada de quatro folhosas. Floresta e Ambiente, Seropédica, v. 21, n. 1, p. 99-107, 2014. DOI: http://dx.doi.org/10.4322/floram.2014.008

ESTEVES, B. M.; DOMINGOS, I.; PEREIRA, H. Improvement of technological quality of eucalypt wood by heat treatment in air at $170-200{ }^{\circ} \mathrm{C}$. Forest Products Journal, Madison, v. 57, n. 1, p. 47-52, 2007.
MENDES, R. F.; BORTOLETTO JÚNIOR, G.; ALMEIDA, N. F. de; SURDI, P. G.; BARBEIRO, I. N. Effects of thermal pre-treatment and variables of production on properties of OSB panels of Pinus taeda. Maderas. Ciencia y Tecnología, Concepción, v. 15, n. 2, p. 141-152, 2013. DOI: http:/ /dx.doi.org/10.4067/S0718-221X2013005000012 FIGUEROA, M. J. M.; DE MORAES, P. D. Comportamento da madeira a temperaturas elevadas. Ambiente Construído, Porto Alegre, v. 9, n. 4, p. 157-174, 2009.

GLASS, S. V.; ZELINKA, S. L. Moisture relations and physical properties of wood. Wood handbook: wood as an engineering material: chapter 4 . Centennial ed. General technical report FPL; GTR-190. Madison, WI: US Dept. of Agriculture, Forest Service, Forest Products Laboratory, 2010. 19p.

GUNDUZ, G; AYDEMIR, D.; KARAKAS, G. The effects of thermal treatment on the mechanical properties of wild Pear (Pyrus elaeagnifolia Pall.) wood and changes in physical properties. Materials and Design, Surrey, v. 30, p. 43914395, 2009. DOI: https://doi.org/10.1016/j.matdes.2009.04.005

HE, Z.; QU, L.; WANG, Z.; QIAN, J.; YI, Y. Effects of zinc chloride-silicone oil treatment on wood dimensional stability, chemical components, thermal decomposition and its mechanism. Scientific Reports, v. 9, e1601, 2019. DOI: https://doi.org/10.1038/s41598-018-38317-5

HILL, C. Wood modification: chemical, thermal and other processes. 1ed. West Sussex: John Wiley \& Sons, 2006. 239p.

LENGOWSKI, E. C.; MUÑIZ, G. I. B.; KLOCK, U.; NISGOSKI, S. Potential use of nir and visible spectroscopy to analyze chemical properties of thermally treated wood. Maderas, Ciencia y Tecnología, Concepción, v. 20, n. 4, p. 627-640, 2018. DOI: http://dx.doi.org/10.4067/S0718-221X2018005041001

LIMA, M. D. A segregação de resíduos do manejo florestal sustentável para otimização da produção bioenergética na Amazônia brasileira. 2020. 206 f. Dissertação (Mestrado em Ciências Florestais) Universidade Federal Rural da Amazônia, Belém, 2020.

PONCSÁK, S.; KOCAEFE, D.; BOUAZARA, M.; PICHETTE, A. Effect of high temperature treatment on the mechanical properties of birch (Betula papyrifera). Wood Science and Technology, New York, v. 40, n. 8, p. 647-663, $2006 . \quad$ DOI: https://doi.org/10.1007/s00226-006-0082-9

POUBEL, D.; GARCIA, R. A.; DOS SANTOS, W. A.; OLIVEIRA, G.; ABREU, H. Efeito da termorretificação nas propriedades físicas e químicas da madeira de Pinus caribaea. Cerne, Lavras, v.19, n. 3, p. 391-398, 2013. DOI: https://doi.org/10.1590/S010477602013000300005

REIS, A. R. S. Anatomia da madeira de quatro espécies de Aspidosperma Mart. \& Zucc. Comercializadas no estado do Pará, Brasil. Ciência da Madeira, Pelotas, v. 6, n. 1, p. 47-62, 2015.2 DOI: http://dx.doi.org/10.15210/cmad.v6i1.5444

SLIK, J. W. F.; RODRÍGUEZ, A. V.; AIBA, S. I.; ALVAREZ, L. P.; ALVES, L. F., ASHTON, P.; BERNACCI, L. An estimate of the number of tropical tree species. Proceedings of the National Academy of 
Sciences, Washington, v. 112, n. 24, p. 7472-7477, 2015. DOI: https://doi.org/10.1073/pnas.1423147112

STALLBAUN, P. H.; BARAUNA, E. E. P.; PAES, J. B.; RIBEIRO, N. C.; MONTEIRO, T. C.; ARANTES, M. D. C. Resistência natural da madeira de Sclerolobium paniculatum Vogel a cupins em condições de laboratório. Floresta e Ambiente, Seropédica, v. 24, e20160013, 2016. DOI: https://doi.org/10.1590/21798087.001316

SUCHY, M; KONTTURI, E.; VUORINEN, T. Impact of drying on wood ultrastructure: similarities in cell wall alteration between native wood and isolated wood-based fibers. Biomacromolecules, Washington, v. 11, n. 8, p. 2161-2168, 2010.2 DOI: https://doi.org/10.1021/bm100547n

THYBRING, E. E.; THYGESEN, L. G.; BURGERT, I. Hydroxyl accessibility in wood cell walls as affected by drying and re-wetting procedures. Cellulose, Bucharest, v. 24, n. 6, p. 2375-2384, 2017. DOI: https://doi.org/10.1007/s10570-017-1278-x

WATKINS, D.; NURUDDIN, M.; HOSUR, M.; TCHERBI-NARTEH, A.; JEELANI, S. Extraction and characterization of lignin from different biomass resources. Journal of Materials Research and Technology, Rio de Janeiro, v. 4, n. 1, p. 26-32, 2015 DOI: https://doi.org/10.1016/j.jmrt.2014.10.009 